\title{
ANTHOPHORIDAE (HYMENOPTERA; APOIDEA) E FLORA ASSOCIADA EM UMA FORMAÇÃO DE CERRADO NO MUNICÍPIO DE BARREIRINHAS, MA, BRASIL. ${ }^{1}$
}

\author{
Patrícia M.C. ALBUQUERQUE² ${ }^{2}$ José Angelo C. MENDONÇA ${ }^{2}$
}

\begin{abstract}
RESUMO - Os cerrados maranhenses estão localizados na Bacia Parnaibana principalmente ao leste e sul do estado. A área de estudo localiza-se na Mesorregião Oeste do Maranhão entre os municípios de Urbano Santos e Barreirinhas. As coletas foram realizadas mensalmente de setembro de 1991 a agosto de 1992 com 12 horas de duração cada. Foram capturados 196 espécimens, 17 espécies e 6 gêneros de Anthophoridae. Centris foi o gênero com maior diversidade e abundância ( 7 espécies; 115 indivíduos), seguido de Xylocopa $(4 ; 59)$ e Paratetrapedia $(3 ; 17)$. Estiveram em atividade durante o ano todo, porém os maiores picos de abundância no número de indivíduos foram nos meses de outubro e novembro, que coincidiram com o período de maior floração do cerrado. Leguminosae e Malpighiaceae constiuem-se nas familias de plantas mais visitadas pelos Anthophoridae em Barreirinhas.
\end{abstract}

Palavras-chave: Apoidea, Anthophoridae, Abelhas, Cerrado.

Anthophoridae (Hymeonoptera, Apoidea) and their Associated Flora in a "Cerrado" Area in Barreirinhas, Ma, Brazil.

SUMMARY - The "cerrados" of Maranhão are located in the basin of Parnaiba mainly in the eastern and southern part of the state. The area of the study is located in the Western Mesorregion of Maranhão between the municipalities of Urbano Santos and Barreirinhas. The collects were realized monthly from September of 1991 to August of 1992, each one with 12 hours. A total of 196 specimens of Anthophoridae were captured, distributed in 17 species and 6 genus. Centris. was the genus with the biggest diversity and abundance ( 7 species; 115 individuals), followed by Xylocopa $(4 ; 59)$ and Paratetrapedia $(3 ; 17)$. The Anthophoridae visited flowers during all the year, but the biggest abundance in the number of individuals was during the months of October and November, coinciding with the period of larger blooming in the "cerrado". Leguminosae and Malpighiaceae constitute the families of plants more visited by Anthophoridae in Barreirinhas.

Key words: Apoidea, Anthophoridae, bees, "cerrado".

\section{INTRODUÇÃO}

Muito se conhece dos aspectos fisionômicos e físiográficos do cerrado brasileiro, porém pouco se sabe dos aspectos biológicos, principalmente sobre a relação entre sua fauna e flora. Segundo dados de SILBERBAUER-
GOTTSBERGER \& GOTTSBERGER (1988), para as áreas de cerrado nos estados de São Paulo, Mato Grosso e Minas Gerais, cerca de $75 \%$ das espécies vegetais são polinizadas de forma exclusiva, primária ou secundariamente, por abelhas.

Mas, apenas no final da década

Trabalho feito com auxílio financeiro do Conselho Nacional de Desenvolvimento Científico e Tecnológico - CNPq, bolsa de Iniciação Científica nº 800717/91-0 e proj. $n^{\circ}$ 400675/91-9; da Fundação de Amparo à Pesquisa do Estado do Maranhão - FAPEMA, proj. $\mathrm{n}^{\circ} 119 / 92$ DC e da Companhia Vale do Rio Doce - CVRD.

2 Universidade Federal do Maranhão. Departamento de Biologia, Largo dos Amores, 21. Departamento de Biologia da Universidade Federal do Maranhão - UFMA. 
de 70 começaram os estudos com população de abelhas nos cerrados brasileiros, destacando-se os de PEREIRANORONHA \& GOTTSBERGER (1980) e PEREIRA-NORONHA et al (1982) sobre a polinização de algumas espécies de plantas do cerrado em São Paulo e alguns que tratam da fauna de Apoidea desse ecossistema (SILVEIRA, $1989 \mathrm{em}$ Paraopeba - MG; MARTINS, 1990 em Lençóis - BA; CARVALHO, 1990 em Uberlândia - MG e PEDRO, 1992 em Cajuru - SP).

Como o cerrado maranhense possui apenas a metade de sua flora comum à do Planalto Central, estando a outra constituída por elementos peculiares e por representantes de espécies regionais (RIZZINI, 1979), é de grande importância a realização deste trabalho, já que trata-se dos primeiros relatos sobre abelhas e flora visitada nos cerrados maranhenses.

Além dos aspectos relacionados à estrutura da comunidade, foi também objetivo deste trabalho fornecer informações sobre a atividade sazonal de algumas espécies de abelhas da família Anthophoridae em uma região de cerrado no Maranhão e sua relação com as plantas do local.

\section{METODOLOGIA}

\section{Área de Estudo}

Esta pesquisa foi realizada na Baisada Oriental Maranhense ( $7^{\circ} 13^{\prime} 16^{\prime \prime} \mathrm{S} ; 44^{\circ} 33^{\prime} 22^{\prime \prime} \mathrm{W}$ ) entre os municípios de Barreirinhas (20 Km) e Urbano Santos (66 Km), distante cerca de $345 \mathrm{Km}$ da capital do Estado, em meio a aproximadamente $25 \mathrm{Km}$ de cerrado ao longo da rodovia MA-225.

A área de coleta possui 01 ha. dividida em 4 setores, com aproximadamente o mesmo tamanho, os quais estão separados por um caminho bastante arenoso e em forma de cruz. A vegetação da área é classificada como "cerrado típico", por apresentar árvores com galhos tortuosos, de troncos lignificados geralmente de porte médio; a camada arbustiva é dominante em toda a área, sendo formada em sua maior parte por arbustos recorrentes; a camada arbórea é formada por árvores de porte médio com dossel aberto típico de cerrado verdadeiro (George Eiten, inf.pessoal).

Apresenta clima tropical megatérmico e úmido, quase subúmido; o total anual de chuvas é geralmente bem elevado (entre 1600 a $1800 \mathrm{~mm}$ ), com duas estações bem definidas pelo regime sazonal: uma estação chuvosa com duração de 7 meses (janeiro a julho) e uma estação seca de agosto a dezembro (IBGE, 1984). Do ponto de vista térmico caracteriza-se por possuir temperaturas geralmente elevadas durante todo o ano, com uma média anual de 26 a $27^{\circ} \mathrm{C}$ (IBGE, 1984).

\section{Amostragem}

As coletas foram feitas por duas pessoas com duração de 12 horas (primeiro dia das 12:00 às 18:00 e das 6:00 às 12:00 no segundo dia), em um intervalo de aproximadamente 30 dias, de setembro de 1991 a agosto de 1992 , totalizando-se 288 horas de amostragem.

As abelhas coletadas visitando ou não flores, eram separadas em sacos 
plásticos, por hora de coleta e número da planta caso fossem coletadas visitando flores. Em laboratório, eram devidamente montadas e etiquetadas e depositadas na coleção da Universidade Federal do Maranhão.

As espécies de abelhas foram identificadas por J.M.F. Camargo. As plantas foram identificadas por C.S.Rosário e J.A.Ratter.

\section{RESULTADOS E DISCUSSÃO}

\section{Composição, Riqueza de espécies e Abundância Relativa}

Foram capturados 196 espécimens de Anthophoridae, 17 espécies e 6 gêneros. Destes, 170 foram coletados em visita às flores, os demais durante o vôo ou próximos aos seus ninhos, Centris foi o gênero com maior diversidade e abundância, 7 espécies e 115 indivíduos, o que converge com os trabalhos de REBÊLO (no prelo), ALBUQUERQUE \& RÊGO (1989), mas diverge em parte dos levantamentos feitos por CARVALHO (1990) e PEDRO (1992) onde Centris apresentou-se com menor frequência se comparado a outros gêneros de Anthophoridae, sobretudo Paratetrapedia e Ceratina .Xylocopa foi o segundo gênero em abundância (quatro espécies; 59 indivíduos), seguido de Paratetrapedia (três espécies; 17 indivíduos), Ptilotopus (uma espécie; três indivíduos), Ceratina e Epicharis (uma espécie; um indivíduo respectivamente).

Centridini foi representada por três gêneros: Centris, Ptilotopus e Epicharis. Centris caxiensis foi a espécie que apresentou maior atividade no cerrado de Barreirinhas sendo capturados 48 indivíduos, seguida por $C$. byrsonimae n.nudum (36) e C. flavifrons (21).

Exomalopsini esteve representada apenas por Paratetrapedia, capturadas visitando principalmente flores de Malpighiaceae, o que era de se esperar já que elas estão entre os maiores polinizadores de plantas desta família (ALBUQUERQUE \& REGG, 1989).

De Xylocopini foram capturadas 4 espécies diferentes, sendo as mais frequentes Xylocopa frontalis e Xylocopa carbonaria .

\section{Flutuação Sazonal}

Foram encontrados Anthophoridae visitando flores durante todo o ano, inclusive no período de chuva (Fig. 1), correspondendo ao padrão geral de distribuição das espécies em áreas tropicais (REBÊLO, no prelo).

Os maiores picos de abundância foram observados nos meses de outubro e novembro, coincidindo com o período de maior floração das plantas mais visitadas: Leguminosae, Malpighiaceae, Ochnaceae, Lecythidaceae, Anacardiaceae e Caryocaraceae (Tab. 1).

No período de janeiro a maio, meses coincidentes com a estação chuvosa, houve uma nítida diminuição de atividade de Anthophoridae influenciado também por uma menor disponibilidade de recursos florais (Fig, 1).

Observa-se também que em junho, julho e agosto quando as Leguminosae e Malpighiaceae começam a florescer, o número de Anthophoridae aumenta vertiginosamente (Fig. 1; Tab. 1).

Não se pode afirmar que os Anthophoridae de Barreirinhas tenham 


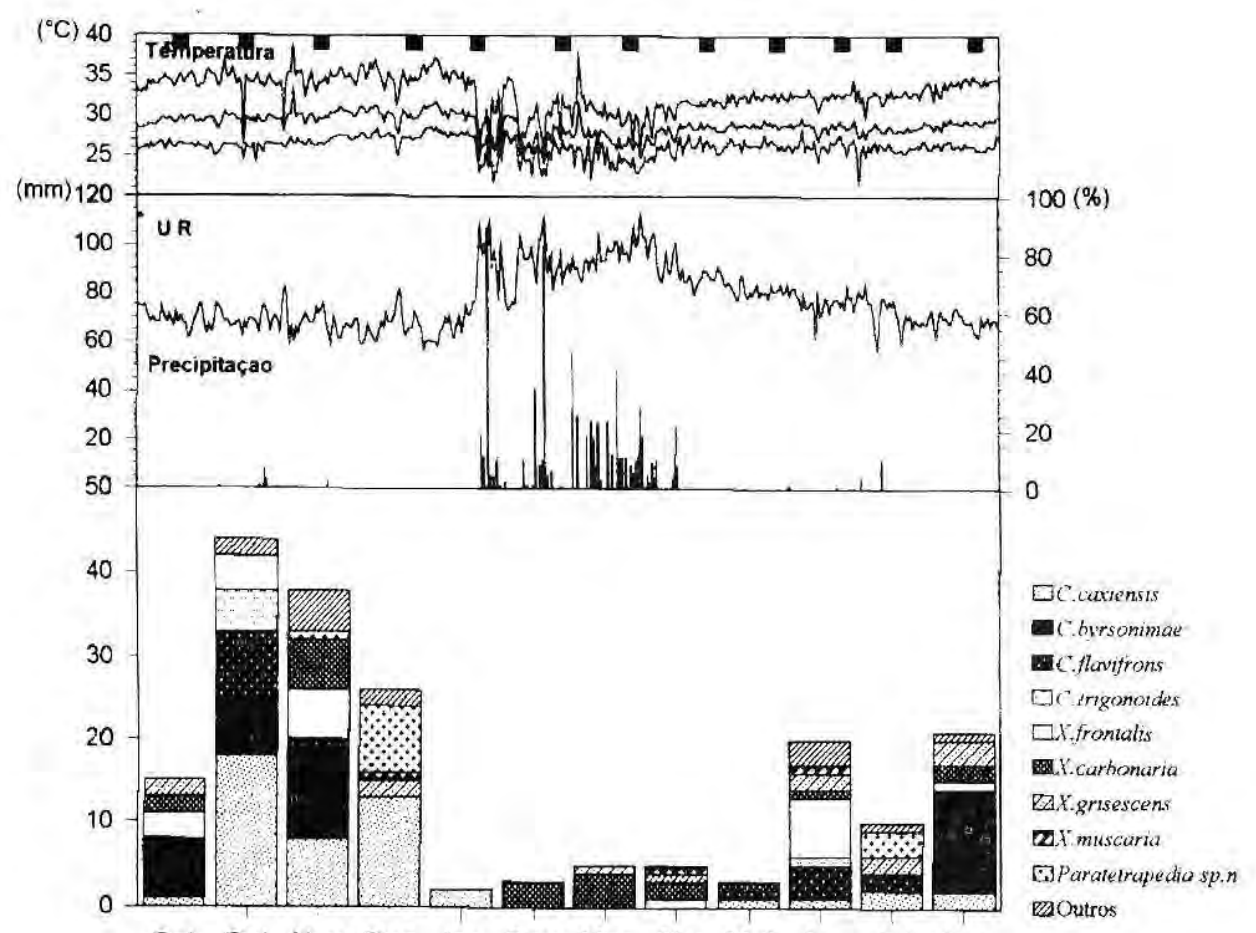

Set Out Nov Dez Jan Fev Mar Abr Mai Jun Jul Ago

Figura 1. Abundância relativa das espécies de Anthophoridae entre Set/91 e Ago/92, em Barreirinhas, MA ( - indica os dias de coleta).

Tabela 1. Número de espécies floridas/famílias de plantas visitadas por Anthophoridae de Set/ 91 a Ago/92 (entre parênteses, a porcentagem de espécies floridas).

\begin{tabular}{|c|c|c|c|c|c|c|c|c|c|c|c|c|}
\hline $\begin{array}{c}\text { Familias de } \\
\text { Plantas }\end{array}$ & SET & OUT & NOV & DEZ & JAN & FEV & MAR & ABR & MAl & JUN & JUL & AGO \\
\hline Anacardiaceae & $02(16,7)$ & $01(2)$ & $01(3)$ & & & & & & & & & \\
\hline Apocynaceae & & & & & & & $03(100)$ & $02(67)$ & & & & \\
\hline Caryocaraceae & & & $01(3)$ & $01(4)$ & & & & & & & & \\
\hline Lecythidaceae & & $05(12)$ & & & & & & $01(33)$ & & & & \\
\hline Leguminosae & $07(58,3)$ & $23(55)$ & $01(3)$ & $02(8)$ & $02(100)$ & & & & & $15(88)$ & $05(56)$ & $05(25)$ \\
\hline Loganiaceae & & & & & & & & & & & & $09(45)$ \\
\hline Malpighiaceae & $03(25)$ & $09(22)$ & $17(59)$ & $23(88)$ & & & & & & $02(12)$ & $04(44)$ & $06(30)$ \\
\hline Ochnaceae & & $04(9)$ & $09(32)$ & & & & & & & & & \\
\hline
\end{tabular}

um período de atividade muito curto no ano, já que as coletas eram feitas apenas uma vez por mês; o que se observou foi que C.caxiensis (capturada em 9 das 12 coletas), $X$. carbonaria (capturada em 8 coletas) e $X$. grisescens (7), apresentaram uma distribuição anual típica de espécies tropicais segundo REBÊLO (1986), SILVEIRA (1989) e PEDRO (1992).

A maior atividade de Centris foi observada em outubro (estação seca), com 5 espécies e 40 indivíduos capturados, sendo C.caxiensis, C.flavifrons e 
C.byrsonimae as espécies mais frequentes (Tab. 2; Fig. 1). Em fevereiro, março e abril (estação chuvosa) não foi coletado nenhum espécimen de Centris; em janeiro, o único indivíduo de C.caxiensis foi coletado próximo ao seu ninho (Fig. 1).

Xylocopa apresentou uma distribuição irregular por todo o ano; X.carbonaria mostrou uma maior atividade no mês de novembro, enquanto $X$.frontalis esteve mais ativa em junho; $X$. grisescens e $X$. muscaria foram bastante irregulares em suas visitas às flores,com poucos indivíduos capturados a cada mês (Fig. 1; Tab. 2).

As três espécies de Paratetrapedia foram observadas nos meses de novembro, dezembro e julho, período coincidente com a floração das Malpighiaceae (Tab. 1 e 2).

Os três espécimens de Ptilotopus maranhensis foram capturados em junho e os únicos exemplares de Ceratina gossypi e Epicharis bicolor foram coletados em dezembro e novembro respectivamente (Tab. 2).

Com relação à influência de umidade relativa e precipitação, observase que quando há um aumento desses dois parâmetros há uma diminuição no número de abelhas em atividade, já a temperatura parece não influenciar diretamente nas atividades dos Anthophoridae, já que as variações observadas a cada mês foram muito pequenas (Fig. 1).

\section{Atividade Diária}

Os horários de maiores frequências são observados no período da manhã (Tab. 3). Os Centridini, coletados principalmente no período da manhã,

Tabela 2. Frequência mensal das espécies de Anthophoridae, entre set/91 e ago/92, em Barreirinhas - MA.

\begin{tabular}{|c|c|c|c|c|c|c|c|c|c|c|c|c|c|}
\hline \multirow{2}{*}{$\begin{array}{r}\text { ABEUHAS } \\
\text { ANTHOPHOAIDAE }\end{array}$} & \multirow[t]{2}{*}{ SET } & \multirow[t]{2}{*}{ at } & \multirow[t]{2}{*}{ NOV } & \multirow[t]{2}{*}{ DEZ } & \multirow[t]{2}{*}{ JAN } & \multirow[t]{2}{*}{ FEV } & \multirow[t]{2}{*}{ MAR } & \multirow[t]{2}{*}{$\mathrm{ABR}$} & \multirow[t]{2}{*}{ MA } & \multirow[t]{2}{*}{ JUN } & \multicolumn{3}{|c|}{ UL AGO Total } \\
\hline & & & & & & & & & & & & & \\
\hline ANTHOPHORINAR & & & & & & & & & & & & & \\
\hline CENTRIDINI & & & & & & & & & & & & & \\
\hline 01. Centris (Centris) caxiensis (Ducke) & 1 & 18 & 8 & 13 & 2 & & & & 1 & 1 & 2 & 2 & 48 \\
\hline Q2. Centris (Paremisia) byrsonimae Moure in litt. & 6 & 7 & 11 & & & & & & & & & 12 & 36 \\
\hline 03. Centris (Hemisiella) trigonoides Lepeletier & & 5 & & & & & & & & 1 & & & 6 \\
\hline $\begin{array}{l}\text { 04. Centris (Centris) flavifons (Fabricius) } \\
\text { 05. Centris (Centris) soiloooda (Moure) }\end{array}$ & 1 & $\begin{array}{c}12 \\
2\end{array}$ & 1 & & & & & & 1 & 4 & 2 & & $\begin{array}{l}21 \\
2\end{array}$ \\
\hline 06. Centris (Hemisiella) tarsata Smith & & & 1 & & & & & & & & & & 1 \\
\hline 07. Centris (Trachina) longimana Fabricius & & & & & & & & & & & & 1 & 1 \\
\hline 08. Pilotopus (Ptilotopus) mararhensis & & & & & & & & & & 3 & & & 3 \\
\hline 09. Epicharis (Xanthepicharis) bicolor Smith & & & 1 & & & & & & & & & & 1 \\
\hline EXOMALOPSINI & & & & & & & & & & & & & \\
\hline 10 Paratetrapedia (Amphipedia) sp. n. & & & 1 & 8 & & & & & & & 3 & & 12 \\
\hline 11 Paratetrapedia (Xanthopedia) glooulosa(Friese) & & & 1 & & & & & & & & & & 1 \\
\hline 12 Paratetrapedia (Tropidopedia) duckei(Friese) & & & 2 & 1 & & & & & & & 1 & & 4 \\
\hline XYLOCOPINI & & & & & & & & & & & & & \\
\hline 13 Xylocopa (Neoxylocopa) cartonana Smith & 2 & & 6 & & & 3 & 4 & 2 & 1 & 1 & & 2 & 21 \\
\hline 14 Xylocopa (Megaxylocopa) frontalis (Oliver) & 3 & 4 & 6 & & & & & 1 & & 7 & & 1 & 22 \\
\hline 15 Xylocopa (Neoxylocopa) grisescens Lepeletier & 2 & & & 2 & & & 1 & 1 & & 2 & 2 & 3 & 13 \\
\hline 16 Xylocopa muscaria (Fabricius) & & & & 1 & & & & 1 & & 1 & & & 3 \\
\hline CEAATININI & & & & & & & & & & & & & \\
\hline 17 Ceratina (Crewella) gossypi Schrottky & & & & 1 & & & & & & & & & 1 \\
\hline Total & 15 & 48 & 38 & 26 & 2 & 3 & 5 & 5 & 3 & 20 & 10 & 21 & 196 \\
\hline
\end{tabular}


apresentam-se mais ativos entre 10:00 e 11:00 horas (Tab. 3), o que se assemelha aos resultados de ALBUQUERQUE \& REGGO (1989) cujas espécies de Centris mostraram-se mais ativas no período entre 8:00 e 10:00 hs. Xylocopa esteve mais ativa entre 7:00 e 9:00 hs.

GOTTSBERGER et al.(1988) observaram que a frequência de visitas de abelhas às flores nas dunas diminuia bastante no final da manhã e à tarde, quando a temperatura se eleva, o que parece uma regra em ambientes com altas temperaturas como também é o cerrado. possui o maior número de espécies com adaptações para coleta de óleo nas flores desta família (BUCHMANN, 1987). Visitaram ainda com certa frequência as espécies de Leguminosae e com menor regularidade Ouratea hexasperma (Ochnaceae), Antonia ovata (Loganiaceae) e Anacardium microcarpum (Anacardiaceae) (Tab. 4, Fig. 2).

Mais de $80 \%$ das Paratetrapedia foram capturadas visitando Malpighiaceae (Tab. 4, Fig. 2). Paratetrapedia também é especialista na coleta de óleo, sendo juntamente com os Centridini os principais

Tabela 3. Distribuição dos indivíduos de Anthophoridae coletados em cada horário ao longo do dia, no período de set/91 a ago/92, em Barreirinhas, MA.

\begin{tabular}{|c|c|c|c|c|c|c|c|c|c|c|c|c|c|}
\hline ABELHAS & $6: 00$ & $7: 00$ & $8: 00$ & $9: 00$ & $10: 00$ & $11: 00$ & $12: 00$ & $13: 00$ & $14: 00$ & $15: 00$ & 16:00 & $17: 00$ & Total \\
\hline \multicolumn{14}{|l|}{ CENTRIDINI } \\
\hline 01. Centris (Centris) caxiensis & 9 & 7 & 2 & 7 & 10 & 4 & 1 & 1 & 1 & 1 & 4 & 1 & 48 \\
\hline Q. Centris (Paremisia) byrsonimae & 1 & 6 & 2 & 5 & 8 & 8 & 4 & & & 2 & & & 36 \\
\hline Q3. Centris (Hemisiella) trigonoides & & & & & 2 & & 3 & & & 1 & & & 6 \\
\hline 04. Centris (Centris) flavifrons & 2 & & 2 & 1 & 9 & 1 & 1 & & & 1 & 3 & 1 & 21 \\
\hline 05. Centris (Centris) spilopoda & & & & & & & & & & & & & 2 \\
\hline 06. Centris (Hemisiella) tarsata & & & & 1 & & & & & & & & & 1 \\
\hline 07. Centris (Trachina) longimana & & & & & & & & 1 & & & & & 1 \\
\hline Q8. Ptilotopus (Ptilotopus) & & & & & 1 & & & & 1 & 1 & & & 3 \\
\hline 09. Epicharis (Xanthepicharis) bicolor & & & & & & 1 & & & & & & & 1 \\
\hline \multicolumn{14}{|l|}{ EXOMALOPSINI } \\
\hline 10 Paratetrapedia (Amphipedia) sp. n. & & 1 & 2 & & & 2 & & 1 & & 3 & 1 & 2 & 12 \\
\hline 11 Paratetrapedia (Xanthopedia) globulosa & & 1 & & & & & & & & & & & 1 \\
\hline 12 Paratetrapedia (Tropidopedia) duckei & & & & 1 & & 1 & & 2 & & & & & 4 \\
\hline \multicolumn{14}{|l|}{ XYLOCOPINI } \\
\hline 13 Xylocopa (Neoxylocopa) carbonaria & 3 & 5 & 4 & 3 & 3 & 1 & & & & 1 & & 1 & 21 \\
\hline 14 Xylocopa (Megaxylocopa) frontalis & 4 & 2 & 2 & 7 & & 1 & & 3 & 1 & 1 & & 1 & $\mathbb{2}$ \\
\hline 15 Xylocopa (Neoxylocopa) grisescens & 1 & 5 & 3 & & & 1 & & & & 1 & 1 & 1 & 13 \\
\hline 16 xylocopa muscaria & & & 1 & & & 1 & & 1 & & & & & 3 \\
\hline \multicolumn{14}{|l|}{ CERATININI } \\
\hline 17 Ceratina (Crewella) gossypi & & & & & & 1 & & & & & & & 1 \\
\hline Total & 20 & 27 & 18 & 25 & 34 & 22 & 10 & 9 & 3 & 12 & 9 & 7 & 196 \\
\hline
\end{tabular}

\section{Visita às Flores}

Centridini visitou principalmente espécies de Malpighiaceae (Tab. 4, Fig. 2), o que já era esperado, já que esta tribo polinizadores de Malpighiaceae (BUCHMANN, 1987; ALBUQUERQUE \& RÊGO, 1989; CRONQUIST, 1991 apud CARVALHO, 1990). Visitaram 


\section{Centris}

\section{Ptilotopus}

Epicharis

Paratetrapedia

Yylocopa

TOTAL
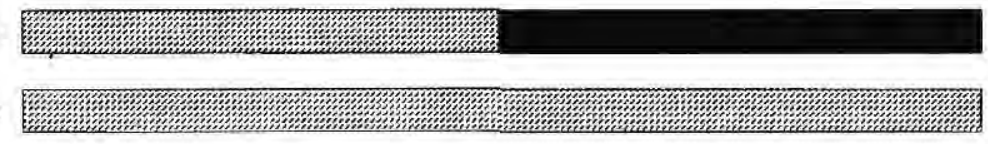

(1)

0

20

40

60

80

100

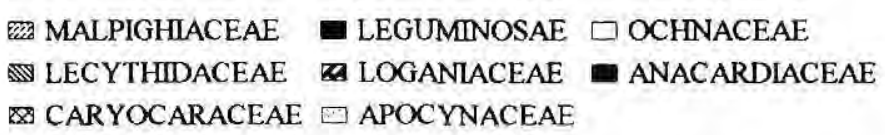

Figura 2. Porcentagem de indivíduos de Anthophoridae nas familias de plantas visitadas no cerrado de Barreirinhas, MA, entre Set/91 e Ago/92.

também Ouratea hexasperma e Anacardium microcarpum (Tab. 4, Fig. 2).

Xylocopa foram as abelhas mais generalistas entre os Anthophoridae, visitando com frequência as espécies de Leguminosae: Vatairea sericea, Cassia sp., Bowdichia sp. e Parkia platycephala, mas também visitaram com uma certa regularidade: Ouratea hexasperma, Eschwellera decolorans (Lecythidaceae), Anacardium microcarpum, Caryocar cuneatum (Caryocaraceae) e Allamanda blancheti (Apocynaceae), demonstrando serem importantes visitantes de plantas do cerrado (Fig. 2).

Um total de 12 espécies florais pertencentes a 8 famílias de plantas foram visitadas poí Anthophoridae no cerrado de Barreirinhas (Tab. 4):

MALPIGHIACEAE: representada por duas espécies no cerrado: Banisteriopsis sp. e Byrsonima amoena; florescendo de junho a dezembro (Tab. 1). Foi uma das famílias mais densamente floridas na área, com pico de floração em outubro e novembro. Foram visitadas principalmente por Centris e Paratetrapedia.

LEGUMINOSAE: representada por 4 espécies - Vatairea sericea ("angelim"), Cassia sp., Bowdichia sp. e Parkia platycephala. Foram visitadas sobretudo por Centris e Xylocopa e um exemplar de Ptilotopus maranhensis. São as espécies de maior distribuição no cerrado e as mais densamente floridas, principalmente em setembro e outubro.

OCHNACEAE: representada apenas por Ouratea hexasperma, com ampla distribuição no cerrado e período de floração de outubro a dezembro (Tab. 1). Visitada principalmente por Xylocopa e com menor frequência por Centris e Paratetrapedia.

LECYTHIDACEAE: apenas Eschwellera decolorans esteve presente no cerrado de Barreirinhas. Floresceu em outubro e abril, com ampla distribuição no cerrado,porém com poucas flores por indivíduo. Foi visitada 
Tabela 4. Espécies florais, número de indivíduos e espécies de abelhas visitantes (veja código na Tab. 2); o número entre parênteses indica o número de indivíduos coletados de cada espécie de abelha.

\begin{tabular}{|c|c|c|c|}
\hline & Espécies de plantas & $\begin{array}{c}\text { Número total } \\
\text { de abelhas } \\
\text { visitantes }\end{array}$ & Espécies de abelhas visitantes \\
\hline \multicolumn{4}{|c|}{ MALPIGHIACEAE } \\
\hline 001 & Banisteriopsis sp. & 50 & $\begin{array}{l}01(11), 02(19), 04(3), 05(1), 07(1), \\
08(1), 09(1), 10(11), 12(2)\end{array}$ \\
\hline \multirow[t]{2}{*}{002} & $\begin{array}{l}\text { Byrsonima amoena } \\
\text { Cuatr. }\end{array}$ & 15 & $01(13), 11(2)$ \\
\hline & LEGUMINOSAE & & \\
\hline 003 & Vatairea sericea Ducke & 55 & $\begin{array}{l}01(15), \quad 02(4), \quad 04(11), \quad 03(5), \quad 05(1), \\
08(1), 13(2), 14(10), 15(4), 16(2)\end{array}$ \\
\hline 004 & Cassia sp. & 5 & $13(1), 14(1), 15(3)$ \\
\hline 005 & Bowdichia sp. & 2 & $01(1), 15(1)$ \\
\hline \multirow[t]{2}{*}{006} & $\begin{array}{l}\text { Parkia platycephala } \\
\text { Benth }\end{array}$ & 2 & $15(2)$ \\
\hline & OCHNACEAE & & \\
\hline \multirow[t]{2}{*}{007} & $\begin{array}{l}\text { Ouratea hexasperma (St. } \\
\text { Hill) Baillon }\end{array}$ & 17 & $\begin{array}{l}01(4), 04(1), 06(1), 11(1), 13(3), 14(6), \\
15(1)\end{array}$ \\
\hline & LECYTHIDACEAE & & \\
\hline \multirow[t]{2}{*}{008} & Eschweilera decolorans & 6 & $04(1), 13(1), 14(4)$ \\
\hline & LOGANIACEAE & & \\
\hline \multirow[t]{2}{*}{009} & Antonia ovata Pohl. & 8 & $02(8)$ \\
\hline & ANACARDIACEAE & & \\
\hline \multirow[t]{2}{*}{010} & $\begin{array}{l}\text { Anacardium microcarpum } \\
\text { Ducke }\end{array}$ & 5 & $02(3), 12(1), 13(1)$ \\
\hline & CARYOCARACEAE & & \\
\hline \multirow{2}{*}{011} & Caryocar cuneatum Witt & 3 & $13(2), 15(1)$ \\
\hline & APOCYNACEAE & & \\
\hline 012 & Allamanda blanchetii DC. & 2 & $13(1), 15(1)$ \\
\hline
\end{tabular}

por Xylocopa e Centris.

ANACARDIACEAE: representada por Anacardium microcarpum, conhecida como "cajuí". Floresceu de setembro a novembro, sendo visitada por Centris, Paratetrapedia e Xylocopa.

LOGANIACEAE: Antonia ovata. Floresceu em agosto, com poucos indivíduos floridos na área de coleta, porém cada indivíduo densamente florido. Visitada somente por Centris. CARYOCARACEAE: Caryocar cuneatum, conhecida como "piqui". Esteve florida em novembro e dezembro com muitos especimens na área, sendo difícil a coleta em suas flores pois são árvores de grande porte. Foi visitada apenas por Xylocopa.

APOCYNACEAE: Allamanda blancheti. Floresceu em março e abril 
(Tab. 1), tem larga distribuição no cerrado, apresentando porém apenas uma flor por indivíduo. Foi visitada apenas por Xylocopa carbonaria e $X$. grisescens.

\section{CONCLUSÕES}

A família Anthophoridae foi relativamente bem representada no cerrado de Barreirinhas com 17 espécies, o que jâ era esperado por tratar-se de um grupo de abelhas tipicamente tropical; porém comparando-se a outras áreas de cerrado no Sudeste e Sul do país, observa-se uma menor diversidade em Barreirinhas. Centridini (grupo de abelhas com maior número de espécies especialistas na coleta de óleo) foi a tribo melhor representada no cerrado, devendo-se isto principalmente à larga distribuição de Malpighiaceae na área de coleta.

As espécies mais frequentes no cerrado são: Centris caxiensis, Centris byrsonimae, Xylocopa frontalis e $X$. carbonaria; todas, com exceção de $C$. byrsonimae estiveram em atividade durante grande parte do ano (cerca de 8 meses).

Leguminosae foi a família com maior distribuição e densidade no local, apresentando sempre pólen e néctar em abundância sendo talvez por esses motivos as mais visitadas por abelhas, principalmente por Xylocopa.

\section{AGRADECIMENTOS}

Os autores agradecem ao Prof. Dr. João M.F, de Camargo pela identificação das abelhas, ao Prof. Dr. James A. Ratter e Carlos da Silva Rosario pela identificação das plantas; a Ciclene Brito pela ajuda nas coletas de campo.

\section{Bibliografia citada}

ALBUQUERQUE, P.M.C. \& RÊGO, M.M.C. 1989. Fenologia das espécies visitantes de murici (Byrsonima crassifolia, MALPIGHIACEAE). Bol. Mus. Para. Emílio Goeldi, zool. Belém. 5(2):163-178.

BUCHMANN, S.L. 1987. The ecology of oil flowers and their bees. Ann.Rev.Ecol.Syst. 18: 343-369.

CARVALHO, A.M.C. 1990. Estudo das interações entre a apifauna e a flora apícola em vegetação de cerrado - Reserva Ecológica do Panga-Uberlândia-MG. FFCLRP-USP, 125p. Dissertação de Mestrado.

GOTTSBERGER, G.; CAMARGO, J.M.F. \& SILBERBAUER-GOTTSBERGER, I. 1988. A bee tropical community: the beach dune vegetation of Ilha de São Luís, Maranhão, Brasil. Bot. Jahrb. Syst., 109(4): 459-500.

INSTITUTO BRASILEIRO DE GEOGRAFIA E ESTATÍSTICA - IBGE. 1984. Coleção de monografias municipais, Rio de Janeiro.

MARTINS, C.F. 1990. Estrutura da comunidade de abelhas (Hym., Apoidea) na caatinga (Casa Nova, BA) e na Chapada Diamantina (Lençóis, BA). Universidade de São Paulo, SP. 159p. Tese de Doutorado.

PEDRO, S.R.M. 1992. Sobre as abelhas (Hym, Apoidea) em um ecossistema de cerrado (Cajuru, NE de estado de São Paulo): composição, fenologia e visita às flores. FFCLRP-USP, SP, 200p. Dissertação de Mestrado.

PEREIRA-NORONHA, M.R.; GOTTSBERGER, G. 1980. A polinização de Aspilia floribunda (Asteraceae) e a relação das abelhas visitantes com outras plantas do cerrado de Botucatu, estado de São Paulo. Rev.Bras.Bot., 3:67-77:

PEREIRA-NORONHA, M.R.; SILBERBAUERGOTTTSBERGER, I.; GOTTSBERGER, G. 1982. Biologia floral de Stylosanthes (Fabaceae) no cerrado de Botucatu estado de Sāo Paulo. Rev.Bras.Biol., 42(3): 595-605.

REBELO, J.M.M.Espécies de Anthophoridae (Hym; Apoidea) e sua associação com flores numa área restrita da Jlha de São Luís, MA, Brasil. Bol.Mus.Para.Emílio Goeldi. No prelo. 
RIZZINI, C.T. 1979. Tratado de fitogeografia do Brasil, Hucitec, ed, da Universidade de São Paulo, v.2, 374p.

SILBERBAUER-GOTTSBERGER, I.; GOTTSBERGER, G. (1988). A polinização das plantas do cerrado. Rev.Bras.Biol, 48(4):651-663,
SILVEIRA, FA. 1989. A fauna de abelhas silvestres e suas fontes de alimento no cerrado de Paraopeba,MG, Viçosa, Universidade Federal de Viçosa, 51p. Dissertação de Mestrado. 